Tropical Journal of Pharmaceutical Research July 2018; 17 (7): 1317-1324

ISSN: $1596-5996$ (print); 1596-9827 (electronic)

(C) Pharmacotherapy Group, Faculty of Pharmacy, University of Benin, Benin City, 300001 Nigeria.

Available online at http://www.tjpr.org

Original Research Article

http://dx.doi.org/10.4314/tjpr.v17i7.13

\title{
Purification, compositional analysis and antioxidant properties of polysaccharides from black ginseng
}

\author{
Li-Hong Gong, Tao Lei, Zhao-Li Zhang, Qi-Chao Liang, Feng-Guo Zhai, Yi-Yan \\ Wu, Xiu-Ping Zhang, Jia-Qi Liu, Jia-Wei Liu* \\ Department of Pharmacology, Mudanjiang Medical University, Mudanjiang City, Heilongjiang Province, 157011, China \\ *For correspondence: Email: jwliu1985@163.com
}

Sent for review: 9 April 2018

Revised accepted: 26 June 2018

\begin{abstract}
Purpose: To extract, purify black ginseng polysaccharides, and study their compositional analysis and antioxidant properties.

Methods: Crude polysaccharides from black ginseng were prepared by hot water extraction and subjected to chromatographic purification on Sephadex G-75 and DEAE-cellulose and Sephadex G-75 columns to yield 4 polysaccharide components: BGP-60, BGP-65, BGP-70 and BGP-80. The BGPS were characterized by chemical analysis, gel permeation chromatography (GPC), gas chromatographymass spectrometry (GC-MS), and Fourier transform-infrared spectroscopy (FT-IR). Finally, the in vitro antioxidant activities of the BGPs were determined through their capacities to scavenge superoxide anion, as well as 1,1-diphenyl-2-picrylhydrazyl (DPPH) and hydroxyl radicals.

Results: The four fractions designated BGP-60, BGP-65, BGP-70 and BGP-80 were polysaccharides with glucose as the main component. They were acidic in nature, with estimated molecular weights (MWs) of 28.6, 26.7, 11.4 and $3.05 \mathrm{kDa}$, respectively. Fractions BGP-60, BGP-65 and BGP-80 had $\alpha$ type glycosidic linkage, whereas BGP-70 had $\beta$-type glycosidic linkage. Compared with vitamin $C$ (vit C), it was found that BGP-60, BGP-65, BGP-70 and BGP-80 had strong potential antioxidant activities; $B G P-60$ exhibited a stronger antioxidant activity than BGP-65, BGP-70 or BGP-80 against DPPH and superoxide anion radicals, while BGP-65 had the highest antioxidant activity against hydroxyl radical. Conclusion: These results suggest that BGPs may be beneficial in the development and manufacture as potential therapeutic agents and functional foods.
\end{abstract}

Keywords: Black ginseng, Polysaccharides, Purification, Antioxidant activity, Functional foods

\begin{abstract}
This is an Open Access article that uses a funding model which does not charge readers or their institutions for access and distributed under the terms of the Creative Commons Attribution License (http://creativecommons.org/licenses/by/4.0) and the Budapest Open Access Initiative (http://www.budapestopenaccessinitiative.org/read), which permit unrestricted use, distribution, and reproduction in any medium, provided the original work is properly credited.

Tropical Journal of Pharmaceutical Research is indexed by Science Citation Index (SciSearch), Scopus, International Pharmaceutical Abstract, Chemical Abstracts, Embase, Index Copernicus, EBSCO, African Index Medicus, JournalSeek, Journal Citation Reports/Science Edition, Directory of Open Access Journals (DOAJ), African Journal Online, Bioline International, Open-J-Gate and Pharmacy Abstracts
\end{abstract}

\section{INTRODUCTION}

The dried roots and rhizome of Panax ginseng were important components of a famous traditional Chinese medicine for thousands of years. Black ginseng is a processed product from fresh ginseng, which is made by repeated steaming and drying [1].
Research on black ginseng is famous in South Korea, but rare in Europe and America. Most of the research are focused on technology, while a few addressed the chemical constituents. In contrast, studies on its polysaccharide content are rare [2]. At present, South Korea is leading the world in the processing and research on black ginseng. Black 
ginseng has appreciable biological activities such as anti-inflammatory, anti-tumor, and immune boosting capacities [3,4]. Some rare ginsenosides with strong anti-cancer effects have been isolated from black ginseng [5]. At present, there are no uniform quality standards for black ginseng polysaccharides. These carbohydrates promote thymus and spleen indices, and they also have immunity-enhancing potential [6]. They activate lymphocyte proliferation or maturation [7], and stimulate cytokine secretion [8]. Their biological activities are closely related to their physicochemical properties and structures [9-11]. The black ginseng used in this experiment was prepared in the laboratory by non -traditional technology.

The present study involved a preliminary characterization, and determination of antioxidant activities of black ginseng polysaccharides (BGPs).

\section{EXPERIMENTAL}

\section{Materials and chemicals}

DPPH radicals, DEAE-cellulose and standard monosaccharides (L-rhamnose, L-arabinose, Dglucose, D-galactose, D-mannose and D-xylose) were products of Sigma (UK). Dextrans of various MWs were Pharmacia products. Other solvents and chemicals used were all of analytical grade.

\section{Extraction and purification of black ginseng polysaccharides}

Powder of black ginseng was extracted successively with ethanol in a reflux unit, and then extracted thrice using 8 volumes of water for $12 \mathrm{~h}$. The aqueous extracts were combined and filtered. Then using a rotary evaporator, the volume of the pooled extract was reduced by nine-tenth. It was subsequently subjected to precipitation for $12 \mathrm{~h}$ at $4{ }^{\circ} \mathrm{C}$ using $80 \%$ ethanol, and clarified by centrifugation for $15 \mathrm{~min}$ at 4000 g. The resultant residue was suspended in water and de-proteinated as described earlier [12]. The aqueous fraction was passed through a 0.22-um molecular membrane and freeze-dried to yield the crude BGP which was re-constituted to $50 \%$ solution with ethanol, and kept refrigerated at $4{ }^{\circ} \mathrm{C}$ for $12 \mathrm{~h}$ to allow for polysaccharide precipitation. Following centrifugation for $15 \mathrm{~min}$ at $4000 \mathrm{~g}$, the supernatant was subjected to successive BGP fractionation using increasing gradients of ethanol i.e. 50 - $60 \%, 60-65 \%$, 65 - $70 \%, 70$ $75 \%$, and $75-80 \%$ ), which resulted in five precipitation fractions. These fractions were subjected to chromatographic purification using Sephadex G-75 and DEAE-cellulose columns, to yield 4 purified fractions coded BGP-60, BGP-65, BGP-70 and BGP-80.

\section{Assays for sulfate, total sugar, uronic acid and protein levels}

The phenol-sulfuric acid method was used to determine the sugar contents of BGP-60, BGP65, BGP-70 and BGP-80, with glucose serving as standard [13], while protein levels were assayed with Bio-Rad Protein Assay [14]. Uronic acid was assayed using sulfuric acid-carbazole method [15], while the barium chloride-gelatin method was employed for the assay for sulfate levels [16]. Bovine serum albumin (BSA), Dglucuronic acid, and potassium sulfate were used as standards in the protein, uronic acid and sulfate assays, respectively.

\section{Estimation of average molecular weights (MWs) of BGPs}

The average MWs of the various BGPs were determined by GPC using a Waters 515 HPLC equipment (Waters Co. Ltd., USA) bearing an ultra-hydrogel column (PL aquagel-OH MIXED; ultra-hydrogel column: length $\times$ aperture: $300 \times 7.5$ $\mathrm{mm}$ ID), with $0.1 \mathrm{M} \mathrm{NaNO}_{3}$ as mobile phase. Using pure water as mobile phase, each sample was analyzed at flow rate, column temperature and injection volume values of $0.6 \mathrm{~mL} / \mathrm{min}, 40$ ${ }^{\circ} \mathrm{C}$, and $20 \mu \mathrm{L}$, respectively, and the MWs were extrapolated from dextran-T standard calibration curves prepared under the same operating conditions. The average MWs were calculated with empower software.

\section{Determination of monosaccharide contents of BGPs}

The monosaccharide contents of the BGPs were determined by GS-MS (QP2010, Shimadzu, Japan) as described previously [17]. Sample (2 $\mathrm{mg}$ ) was subjected to hydrolysis with trifluoroacetate $(2 \mathrm{M})$ for $1 \frac{1}{2} \mathrm{~h}$. The solution was placed in a rotary evaporator and $2 \mathrm{~mL}$ of methanol was added until it was dried up. This was done two times, and the resultant residue was taken up in water (double-distilled) and subjected to borohydride reduction for $8 \mathrm{~h}$. The sodium borohydride was neutralized by addition of acetic acid. Then the mixture was placed in the rotary evaporator and dried by sequential addition of three 3-mL batches of methanol (each $3 \mathrm{~mL}$ added was allowed to dry before the next addition), followed by oven-drying at $110{ }^{\circ} \mathrm{C}$. Acetylation was done by adding $1 \mathrm{~mL}$ of acetic anhydride at $100{ }^{\circ} \mathrm{C}$ for $1 \mathrm{~h}$, and the mixture was cooled by addition of $3 \mathrm{~mL}$ toluene. Thereafter, the product was vacuum-concentrated to dryness 
(this process was repeated 5 times to remove unreacted acetic anhydride). The product was dissolved in $3 \mathrm{~mL}$ chloroform, and fractionated in a separating funnel by addition of $10 \mathrm{~mL}$ distilled water, followed by thorough shaking. The upper aqueous layer was discarded (this operation was carried out 4 times), and the $\mathrm{CHCl}_{3}$ layer was dried with anhydrous sodium sulfate, and subjected to GC-MS analysis, with He as carrier gas at a flow rate of $1.0 \mathrm{ml} / \mathrm{min}$. The detector and injection temperatures were $250{ }^{\circ} \mathrm{C}$. The temperature of the column was increased at $3{ }^{\circ} \mathrm{C}$ intervals from 120 to $250{ }^{\circ} \mathrm{C}$, and maintained at $250{ }^{\circ} \mathrm{C}$ for $5 \mathrm{~min}$.

\section{UV-VIS spectral analysis}

Aqueous solutions of BGP-60, BGP-65, BGP-70 and BGP-80 were separately scanned in the wavelength range of 200- 400 in a Shanghai UVVIS spectrophotometer.

\section{Fourier-transform infrared spectra (FT-IR) analysis}

The samples (BGP-60, BGP-65, BGP-70 and BGP-80) were ground with $\mathrm{KBr}$ powder and pelleted prior to use for FTIR analysis (frequency range: $4000-400 \mathrm{~cm}^{-1}$ ) in a Perkin Elmer Fourier transform IR spectrophotometer [18].

\section{Evaluation of antioxidant activity}

\section{DPPH scavenging assay}

The DPPH scavenging capacities of BGP-60, BGP-65, BGP-70 and BGP-80 were determined by the method of Yang et al [19]. $1 \mathrm{~mL}$ each of different concentrations of polysaccharide solution was incubated with $0.1 \mathrm{mM}$ ethanolic solution of DPPH $(2 \mathrm{~mL})$ at $25{ }^{\circ} \mathrm{C}$ for $20 \mathrm{~min}$, and the absorbance was read at $517 \mathrm{~nm}$. Assays done with distilled water in place of sample served as blank, while vitamin C served as positive control. The absorbance values were used to calculate the DPPH scavenging capacity (D) as in Eq 1.

$D(\%)=\left\{1-\left(A_{1}-A_{2}\right) / A_{0}\right\} 100$

where $A_{0}$ is control absorbance, $A_{1}$ is absorbance of sample and DPPH, and $A_{2}$ is the absorbance of the sample blank. The $\mathrm{IC}_{50}$ values of the samples were extrapolated from the standard curve.

\section{$\mathrm{OH}$ scavenging capacity}

The OH scavenging capacities of BGP-60, BGP65, BGP-70 and BGP-80 were determined as outlined by Chen et al. [20]. A range of concentrations of each BGP (I mL each) was incubated for $30 \mathrm{~min}$ at $37^{\circ} \mathrm{C}$ with $2 \mathrm{ml}$ of $9 \mathrm{mM}$ $\mathrm{FeSO}_{4}, 0.1 \mathrm{~mL}$ of $20 \mathrm{mM} \mathrm{H} \mathrm{O}_{2}, 1 \mathrm{~mL}$ of deionized $\mathrm{H}_{2} \mathrm{O}$ and $1.5 \mathrm{~mL}$ of $9 \mathrm{mM}$ solution of salicylic acid in ethanol. Vitamin $\mathrm{C}$ was used as standard. At the end of reaction, absorbance was read at $510 \mathrm{~nm}$ in a spectrophotometer, and the $\mathrm{OH}$ scavenging ability $(\mathrm{Y})$ obtained using Eq 2.

$\left.Y(\%)=\left\{1-\left(A_{1}-A_{2}\right) / A_{0}\right)\right\} \times 100$

where $A_{0}$ is control absorbance, $A_{1}$ is sample absorbance (with $\mathrm{OH}$ ), and $A_{2}$ is sample blank absorbance. The corresponding $\mathrm{IC}_{50}$ values were extrapolated from a standard curve.

\section{$\mathrm{SO}_{2}^{-}$scavenging capacity}

This was assayed by a slight modification of the procedure described by Han et al [21]. A range of concentrations of each BGP $(0.5 \mathrm{~mL}$ each) was incubated at $25{ }^{\circ} \mathrm{C}$ with $45 \mathrm{mM}$ pyrogallate $(0.1$ $\mathrm{mL}$ ), $0.05 \mathrm{M}$ Tris buffer, $\mathrm{pH} 7.8$, and $0.5 \mathrm{~mL}$ of deionized $\mathrm{H}_{2} \mathrm{O}$ for 20 min. The reaction mixture consisted of $3 \mathrm{~mL} 0.05 \mathrm{M}$ Tris-HCL buffer, $\mathrm{pH}$ 7.8 , and $0.1 \mathrm{~mL}$ pyrogallate $(45 \mathrm{mM}), 0.5 \mathrm{ml}$ of deionized $\mathrm{H}_{2} \mathrm{O}$ ) for $20 \mathrm{~min}$. Then, $0.1 \mathrm{~mL}$ of 45 $\mathrm{mM}$ pyrogallate was added, followed by further incubation for $4 \mathrm{~min}$, after which absorbance was read at $420 \mathrm{~nm}$. Ascorbic acid was used as control. The $\mathrm{SO}_{2}$ scavenging capacity (Q) was obtained for each BGP using Eq 3.

$\left.Q(\%)=\left\{1-\% A_{1}-A_{2}\right) / A_{0}\right\} \times 100$

where $A_{0}$ is control absorbance value, $A_{1}$ is sample absorbance (with $\mathrm{SO}_{2}{ }^{-}$), and $\mathrm{A}_{2}$ is the sample blank absorbance. The corresponding $I_{50}$ values were extrapolated from a standard calibration curve.

\section{Statistical analysis}

Data are presented as mean \pm standard deviation (SD). Differences between BGPs were analyzed statistically with one-way analysis of variance (ANOVA) and Duncan's least significant test. All analyses were carried out using SPSS version 13. Values of $p<0.05$ were taken as indicative of statistical significance.

\section{RESULTS}

\section{Purified BGPs}

Black ginseng polysaccharide was obtained in an overall yield of $17.63 \%$ through water extraction, ethanol precipitation, filtration through $0.22 \mu \mathrm{m}$ molecular membrane, and lyophilization. 
Following chromatographic purification with Sephadex G-75 and DEAE-cellulose, four fractions were obtained: BGP-60, BGP-65, BGP70 and BGP-80. The recoveries of BGP-60, BGP-65, BGP-70 and BGP-80 based on the amount of crude BGPs used were 9.78, 8.65, 14.62 and $4.21 \%$, respectively. The four fractions yielded single, narrow and symmetrical peaks when eluted in GPC, indicating their homogeneity (Figure 1). The GPC analysis indicated that the average MWs of BGP-60, BGP-65, BGP-70 and BGP-80 were approximately 28.6, 26.7, 11.4 and $3.05 \mathrm{kDa}$, respectively. Results from UV-VIS spectrum in the wavelength range of $200-400 \mathrm{~nm}$ showed no absorption from 260 to $280 \mathrm{~nm}$, suggesting the absence of DNA, RNA and proteins.
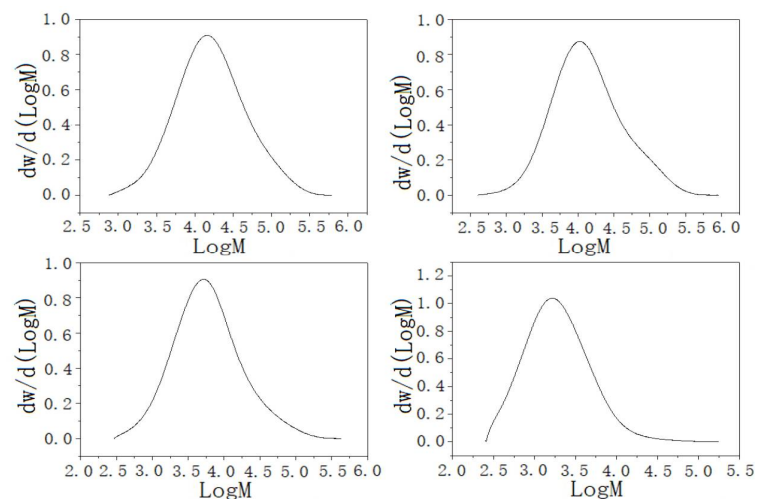

Figure 1: Molecular weights of BGP-60, BGP-65, BGP-70 and BGP-80, as obtained using GPC

\section{Compositions of the BGPs}

The levels of carbohydrate, protein, uronic acid and sulfate in BGP-60, BGP-65, BGP-70 and BGP-80 are shown in Table 1. The carbohydrate contents of BGP-60, BGP-65, BGP-70 and BGP80 were $79,54,78$ and $17 \%$, respectively. None of the four polysaccharide fractions contained proteins. The contents of uronic acid in BGP-60, BGP-65, BGP-70 and BGP-80 were 4, 23, 18 and $63 \%$, respectively, while sulfate contents were 17, 23, 4 and $20 \%$, respectively. These results indicate differences in chemical components of the BGPs.

\section{Monosaccharide composition of BGPs}

The monosaccharide composition of BGP-60, BGP-65, BGP-70 and BGP-80 are presented in Table 2 and Figure 2. BGP-60 contained galactose and glucose in an approximate molar ratio of 22:78, with glucose as the main monosaccharide, while BGP-65, BGP-70 and BGP-80 contained mainly arabinose, galactose and glucose, with glucose also as the predominant monosaccharide.

\section{FT-IR spectra of BGPS}

The FT-IR spectra of BGP-60, BGP-65, BGP-70 and BGP-80 are presented in Figure 3. Fraction BGP-60 had specific absorptions at 3433,2927 , 1635, 1026, 1111, 1153 and $853 \mathrm{~cm}^{-1}$, while BGP-65 had specific absorptions at 3384, 2933, $1636,1043,1078,1151$ and $854 \mathrm{~cm}^{-1}$. Fraction BGP-70 had specific absorptions at 3420, 2927, 1635, 1044, 1078, 1151 and $892 \mathrm{~cm}^{-1}$, while BGP-80 had specific absorptions at 3395, 2928, $1633,1041,1078,1149$ and $860 \mathrm{~cm}^{-1}$.

\section{Antioxidant properties of BGPs}

\section{DPPH radical scavenging capacity}

The DPPH scavenging capacities of BGP-60, BGP-65, BGP-70, BGP-80 were lower than that of vitamin $C$ (Figure 4). The scavenging activities of BGP-60, BGP-65, BGP-70 and BGP-80 at 8 $\mathrm{mg} / \mathrm{mL}$ reached $96.33,81.47,91.07$ and 92.38 $\%$, respectively, with $\mathrm{IC}_{50}$ values of BGP-60, BGP-65, BGP-70 and BGP-80 for 1.80, 3.26, 1.93 and $2.36 \mathrm{mg} / \mathrm{mL}$, respectively.

Table 1: Carbohydrate, protein, uronic acid and sulfate levels in BGP-60, BGP-65, BGP-70, and BGP-80

\begin{tabular}{lcccc}
\hline Sample & Protein (\%) & Carbohydrate (\%) & Uronic acid (\%) & Sulfuric radical (\%) \\
\hline BGP-60 & 0 & 79 & 4 & 17 \\
BGP-65 & 0 & 54 & 23 & 23 \\
BGP-70 & 0 & 78 & 18 & 4 \\
BGP-80 & 0 & 17 & 63 & 20 \\
\hline
\end{tabular}

Table 2: Monosaccharide compositions of the four BGPs

\begin{tabular}{|c|c|c|c|c|c|c|c|}
\hline \multirow[t]{2}{*}{ Sample } & \multicolumn{7}{|c|}{ Sugar content (\%) } \\
\hline & Rhamnose & Fucose & Arabinose & Xylose & Mannose & Glucose & Galactose \\
\hline BGP-60 & - & - & 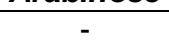 & (1) & - & 77.67 & 22.33 \\
\hline BGP-65 & - & - & 3.72 & - & - & 59.23 & 37.05 \\
\hline BGP-70 & - & - & 5.18 & - & - & 51.43 & 43.39 \\
\hline BGP-80 & - & - & 6.66 & - & - & 51.61 & 41.73 \\
\hline
\end{tabular}



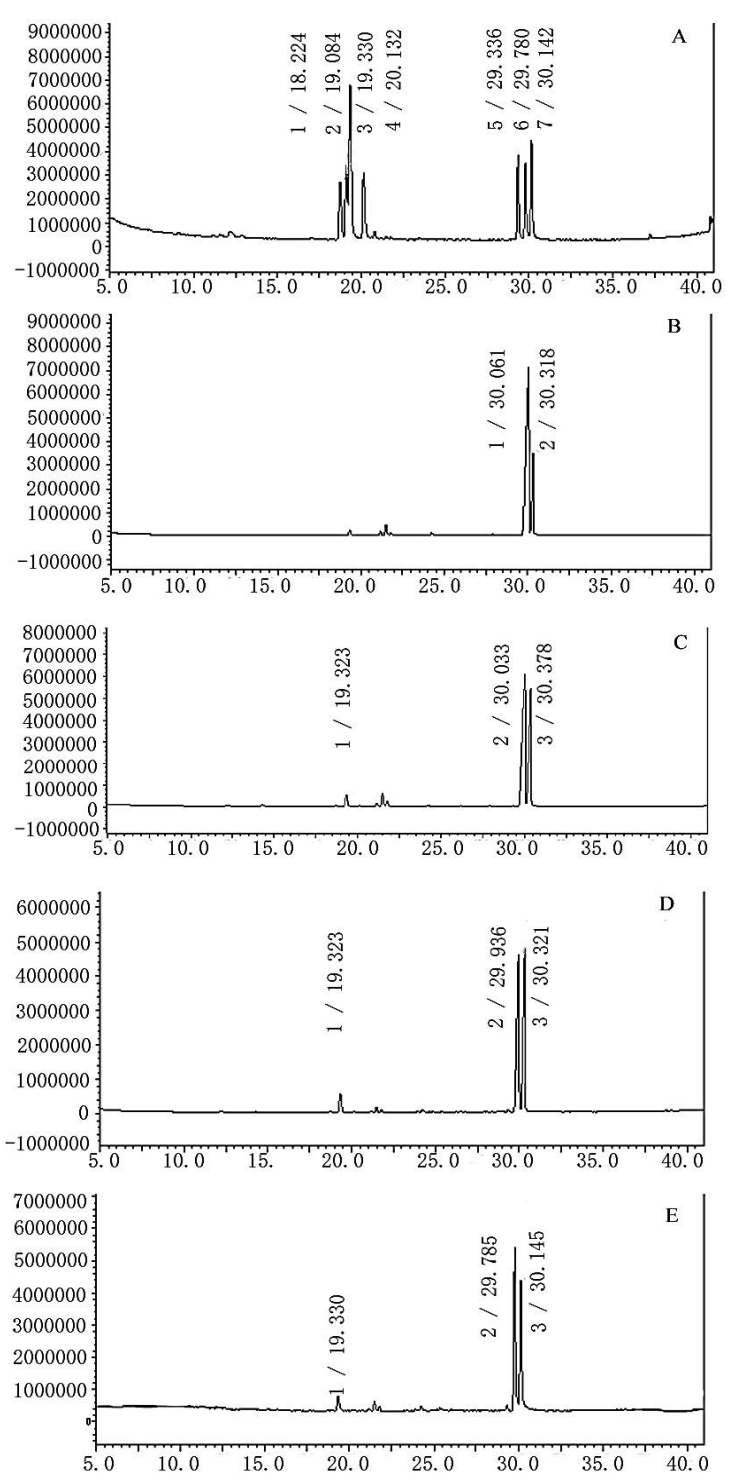

Figure 2: GC-MS chromatograms of monosaccharide standards (A), BGP-60 (B), BGP-65 (C), BGP-70 (D) and BGP-80 (E)

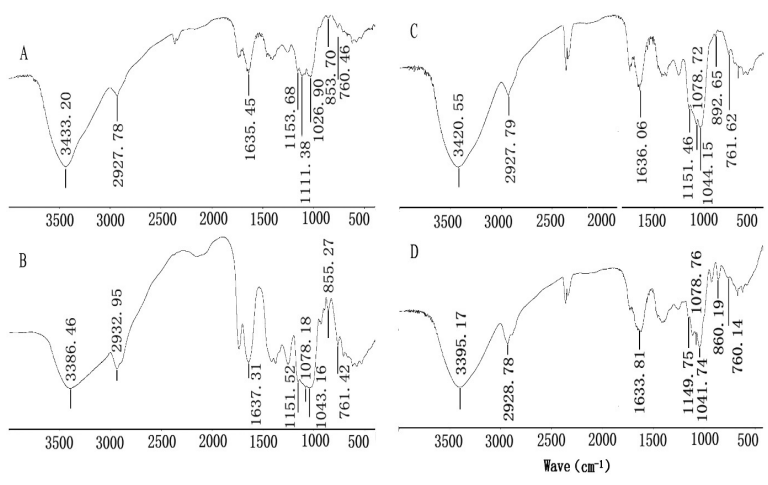

Figure 3: FTIR spectra of BGP-60 (A), BGP-65 (B), BGP-70 (C) and BGP-80 (D)

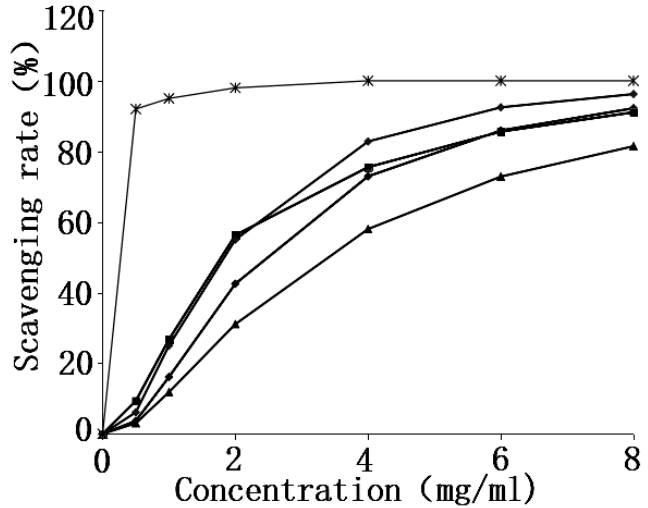

Figure 4: Scavenging effect of BGP-60, BGP-65, BGP-70 and BGP-80 on DPPH radicals $\bullet: B G P-80$, $\mathbf{n}$ : BGP-70, $\mathbf{\Delta}:$ BGP-65, $\bullet: B G P-60, *$ Vitamin $C$

\section{$\mathrm{OH}$ scavenging capacity}

The $\mathrm{OH}^{\bullet}$ scavenging capacities of BGP-60, BGP-65, BGP-70, BGP-80 and vitamin C are shown in Figure 5 . The $\mathrm{OH}^{*}$ scavenging capacities produced by BGP-60, BGP-65, BGP70 and BGP-80 (87.30, 83.28, 87.71 and 90.89 $\%)$, respectively were concentration-dependent below $0.5 \mathrm{mg} / \mathrm{mL}$, although they were lower when compared with vitamin $C$. The $\mathrm{IC}_{50}$ values of BGP-60, BGP-65, BGP-70 and BGP-80 were $2.28,1.90,2.051 .91 \mathrm{mg} / \mathrm{mL}$, respectively, and the $\mathrm{OH}^{\bullet}$ scavenging activity was in the order: BGP-60 < BGP-70 < BGP-80 < BGP-65.

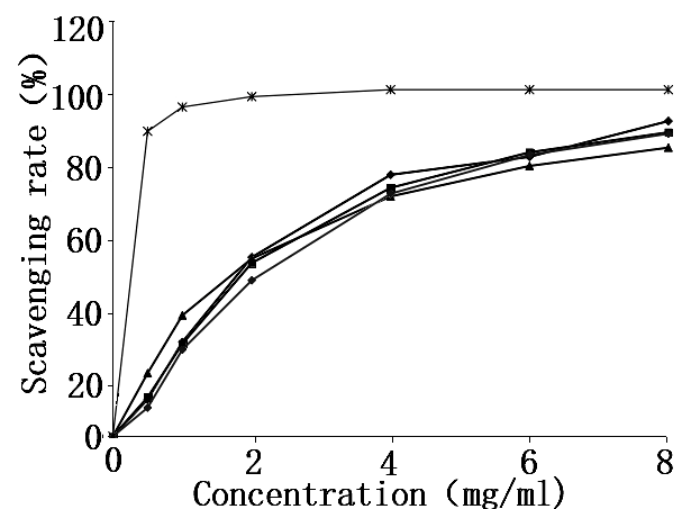

Figure 5: Scavenging effect of BGP-60, BGP-65, BGP-70 and BGP-80 on hydroxyl radical. •: BGP-80, п: BGP-70, $\Delta$ : BGP-65, : BGP-60, *: Vc

\section{$\mathrm{O}_{2}^{-}$scavenging capacity}

Figure 6 shows that the $\mathrm{O}_{2}^{-}$scavenging capacities of BGP-60, BGP-65, BGP-70, and BGP-80 were concentration-dependent below $0.5 \mathrm{mg} / \mathrm{ml}$, although they showed lower scavenging abilities when compared to vitamin C. The potential scavenging activities of BGP-60, BGP-65, BGP-70 and BGP-80 were 83.07, $78.37,77.12$ and $82.97 \%(8 \mathrm{mg} / \mathrm{mL})$, 
respectively; and their $\mathrm{IC}_{50}$ values were 1.95 , $2.88,2.612 .47 \mathrm{mg} / \mathrm{mL}$, respectively.

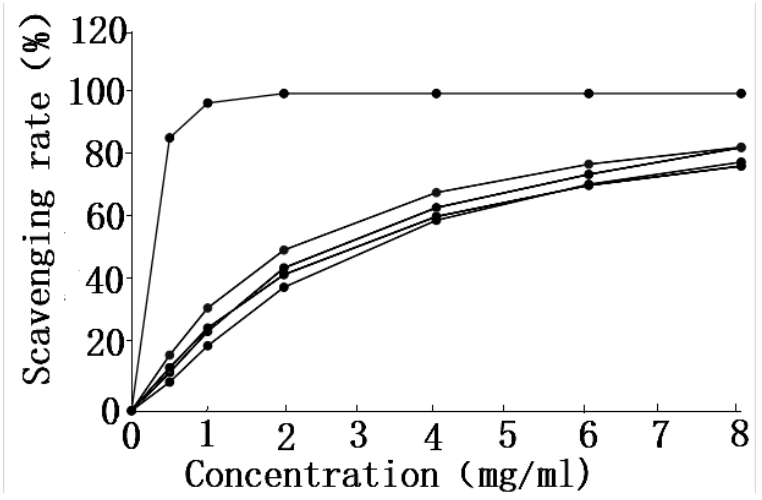

Figure 6: $\mathrm{O}_{2}-$ scavenging effects of BGP-60, BGP-65, BGP-70 and BGP-80. •: BGP-80, - BGP-70, $\mathbf{\Delta}$ : BGP-65, : BGP-60, *: Vitamin C

\section{DISCUSSION}

Black ginseng is a processed product from fresh ginseng. Polysaccharides from black ginseng possess hypoglycemic, anti-tumor, antithrombotic and antioxidant properties. In order to avoid the destruction of the biological activities of these polysaccharides, cold extraction method was used in this study. Free radicals are associated with many diseases. Hydroxyl, DPPH and superoxide radical scavenging activities are widely used for the quantitative determination of antioxidant capacities of biological samples and foods.

The present study reports the isolation and purification of pharmacologically-active polysaccharides from black ginseng. Four major polysaccharide fractions coded BGP-60, BGP65, BGP-70 and BGP-80 were identified. The four polysaccharide fractions showed different degrees of antioxidant activities. The biological effects of polysaccharides are associated with their structural characteristics. Results from GPC analysis indicated wide variations in molecular weights of the BGPs, with values of 28.6, 26.7, 11.4 and $3.05 \mathrm{kDa}$ for BGP-60, BGP-65, BGP-70 and BGP-80, respectively. Monosaccharide compositional analysis showed that BGP-60 consisted of glucose and galactose, while BGP65 , BGP-70 and BGP-80 were contained mainly galactose, arabinose and glucose.

In the infrared spectra analysis, stretches in $\mathrm{OH}$ groups occur within 3600 and $3200 \mathrm{~cm}^{-1}$ (BGP60, BPG-65, BPG-70, and BPG-80 had bands at $3433,3384,3420$, and $3395 \mathrm{~cm}^{-1}$, respectively); while C-H stretching occurs at $2923 \mathrm{~cm}^{-1}$ (BGP60 , BPG-65, BPG-70, and BPG-80 had bands at 2927, 2933, 2927, and $2928 \mathrm{~cm}^{-1}$, respectively).
The $1635 \mathrm{~cm}^{-1}$ is ascribed to bound water (BGP60 , BPG-65, BPG-70, and BPG-80 had bands at 1636,1635 , and $1633 \mathrm{~cm}^{-1}$, respectively) [22].

Each GBP produced a band within the zone $1200-1000 \mathrm{~cm}^{-1}$, which is usually attributed to the overlap between ring vibrations and vibrations due to $(\mathrm{C}-\mathrm{O}-\mathrm{C})$ glycosidic bonds and side groups $(\mathrm{C}-\mathrm{OH})$ [23]. Therefore the absorptions at 1026, 1111 and $1153 \mathrm{~cm}^{-1}$ (for BGP-60); 1043, 1078 and $1151 \mathrm{~cm}^{-1}$ (for BGP65); 1044, 1078 and $1151 \mathrm{~cm}^{-1}$ (for BGP-70); and 1041, 1078 and $1149 \mathrm{~cm}^{-1}$ (BGP-80) indicate a pyranose sugar configuration [24]. The absorptions at $853 \mathrm{~cm}^{-1}$ (BGP-60), $854 \mathrm{~cm}^{-1}$ (BGP-65) and $860 \mathrm{~cm}^{-1}$ (BGP-80) are characteristic absorption peak of $\alpha$-dominating configuration in pyranose sugars [25]. A significant feature peak at $892 \mathrm{~cm}^{-1}$ was seen in BGP-70, revealing the $\beta$-configuration of the sugar units [26]. These results indicate that BGP70 possessed $\beta$-configuration of pyranose ring structure, while BGP-60, BGP-65 and BGP-80 consisted of $\alpha$-dominating configurations in pyranose forms.

At room temperature, DPPH is a stable free radical and it is commonly employed in assessing antioxidant properties of crude extracts and purified fractions. In this study, the ability of the BGPs to scavenge DPPH radicals generally increased in the order: BGP-65 < BGP$80<$ BGP-70 < BGP-60. Iron-chelating compounds which participate in Fenton reaction exert $\mathrm{OH}^{*}$ scavenging capacities [27]. The neutralization of $\mathrm{OH}^{*}$ is extremely important for antioxidant defenses in cells. The $\mathrm{OH}^{\bullet}$ scavenging capacity contributed to the observed antioxidant effects of the BGPs, which increased in the order of BGP-60 < BGP-70 < BGP-80 < BGP-65.

On decomposition, $\mathrm{O}_{2}^{-}$generates $\mathrm{O}^{\bullet}$ and $\mathrm{OH}^{\bullet}$ which are more powerful ROS; these damage lipids, proteins and DNA through deleterious oxidation [28]. Thus, the neutralization of $\mathrm{O}_{2}{ }^{-}$is a critical aspect of all antioxidant investigations. The $\mathrm{O}_{2}$ - scavenging capacities of the BGPs were in the order: BGP-65 < BGP-70 < BGP-80 $<$ BGP-60, and contributed substantially to the overall antioxidant effects of the BGPs.

\section{CONCLUSION}

These results demonstrate that BGPs are potential natural antioxidants that may be used as functional foods. The antioxidant capacities of BGP-60 with respect to DPPH and $\mathrm{O}_{2}{ }^{-}$ scavenging are superior to those of BGP-65, 
BGP-70 or BGP-80; while BGP-65 has the strongest antioxidant activity against $\mathrm{OH}^{\circ}$.

\section{DECLARATIONS}

\section{Acknowledgement}

This work was financed by Research Fund of University Nursing Program for Young Scholars with Creative Talents in Heilongjiang Province (no. UNPYSCT-2017172), Natural Science Foundation of Heilongjiang Province (no. $\mathrm{H} 2016090)$, Research project of Heilongjiang Provincial Health and Family Planning Commission (no.2017-367), both in China.

\section{Conflict of interest}

No conflict of interest is associated with this study.

\section{Contribution of authors}

We declare that this work was done by the author(s) named in this article and all liabilities pertaining to claims relating to the content of this article will be borne by the authors. All authors read and approved the manuscript for publication. Jia-Wei Liu conceived and designed the study, Li-Hong, Tao Lei, Zhao-Li Zhang, QiChao Liang, Feng-Guo Zhai, Yi-Yan Wu, XiuPing Zhang, Jia-Qi Liu collected and analyzed the data, and Li-Hong Gong wrote the manuscript.

\section{REFERENCES}

1. Seo YB. Standard manufacture process of red ginseng by nine-time steaming and nine-time drying. Korea 2010; 40-46.

2. Lee JW. Method of making black ginseng and black ginseng concentrates by successively steaming and aging fresh ginseng 9 times without passing through drying process thereby preventing loss of active saponin. Korea 2015; 08-23.

3. Yuan $Y$, Gan $Y$, Wang F. Study on antitumor effect of the extract from Black ginseng in vivo. J Pract Tradit Chin Med 2015; 31(11): 988-990.

4. Kim SN, Kam SJ. Effects of black (9 times-steaming ginseng) on hypoglycemic action and changes in the composition of ginsenosides on the steaming process. Korean J Food Sci 2009; 41(1): 77-81.

5. Sun BS, Gu LJ, Fang ZM. Simultaneous quantification of 19 ginsenosides in black ginseng developed from Panax ginseng by HPLC-ELSD. J Pharm Biomed Anal 2009; 50(1): 15-22.

6. Bao X, Yuan H, Wang C, Liu J, Lan M. Antitumor and immunomodulatory activities of a polysaccharide from
Artemisia argyi. Carbohydr Polym 2013; 98(1): 12361243.

7. Naeem RF, Amirah I, Zainal AN, Suriza SA. Stimulatory effects of polysaccharide fraction from solanum nigrum on RAW 264. 7 murine macrophage cells. PLOS One 2014; 9(10): e108988.

8. Honda-Okubo Y, Saade F, Petrovsky N. Advax, a polysaccharide adjuvant derived from delta inulin, provides improved influenza vaccine protection through broad-based enhancement of adaptive immune responses. Vaccine 2012; 30(36): 5373-5381.

9. Zhang H, Zhang J, Nan Z. Structure Characterization and Antitumor Activity of Polysaccharide WPP2 from Pleurotus Eryngii. Chem Res Chin Univ 2013; 10(34): 2327-2333.

10. Wang J, Zha X, Pan L. Structural Characterization of an Immunoactive Polysaccharide DNP-W1B from Dendrobium nobile Lindl. Chem Res Chin Univ 2013; 4(34): 881-885

11. Liu-Lei L, Hao X. Extraction, Separation, Structural Analysis and Antioxidant Activity in vitro of Arabinoxylans ( $A X-1$ ) from the Residue of Astragalus Root. Chem Res Chin Univ 2016; 12(37): 2168-2175.

12. Liu Y, Zhang B, Ibrahim SA, Gao SS, Yang H, Huang W. Purification, characterization and antioxidant activity of polysaccharides from Flammulina velutipes residue. Carbohydr Polym 2016; 145: 71-77.

13. Dubois M, Gilles KA, Hamilton JK, Rebers PA, Smith F. Colorimetric determination of sugars and related substances. Anal Chem 1956; 28: 350-356.

14. Bradford MM. A rapid and sensitive method for the quantitation of microgram quantities of protein utilizing the principle of protein-dye binding. Anal Biochem 1976; 72: $248-254$

15. Blumenkrantz N, Asboe-Hansen G. New method for quantitative determination of uronic acids. Anal Biochem 1973; 54: 484-489.

16. Doigson KS, Price RG. A note on the determination of the ester sulfate content of sulfated polysaccharides. Biochem J 1962; 84: 106-110.

17. Ao XL, Xing JH, Song DZ. Purification, composition analysis and antioxidant activity of the polysaccharides from Dendrobium nobile Lindl. Carbohydr Polym 2010; 79: 1014-1019.

18. Yang B, Zhao MM, Shi J, Jiang YM, Yang N. Effect of ultrasonic treatment on the recovery and DPPH radical scavenging activity of polysaccharides from longan fruit pericarp. Food Chem 2008; 106: 685-690.

19. Yan JK, Li L, Wang ZM, Wu JY. Structural elucidation of an exopolysaccharide from mycelial fermentation of a Tolypocladium sp. fungus isolated from wild Cordyceps sinensis. Carbohydr Polym 2010; 79(1): 125-130.

20. Chen $X, W u$ G, Huang Z. Structural analysis and antioxidant activities of polysaccharides from cultured Cordyceps militaris. Int J Biol Macromol 2013; 58: 1822.

Trop J Pharm Res, July 2018; 17(7): 1323 
21. Han SH, Zhu JB, Wang YY. Measurement of the antioxidant activity by pyrogallol autooxidation. China Brewing 2009; 28(6): 155-157.

22. Yu H, Feng, SW. Structural characterization of an active polysaccharide from Phellinus ribis. Carbohydr Polym 2007; 70: 386-392.

23. Shang $M$, Zhang $X$, Dong $Q$, Yao J, Liu Q, Ding $K$. Isolation and structural characterization of the waterextractable polysaccharides from Cassia obtusifolia seeds. Carbohydr Polym 2012; 90: 827-832.

24. Zhao GH, Kan JQ, Li ZX, Chen ZD. Structural features and immunological activity of a polysaccharide from Dioscorea opposita Thunb roots. Carbohydr Polym 2005; 61: 125-131.
25. Barker SA, Bourne EJ, Stacey M, Whiffen DH. Infrared spectra of carbohydrates. Some derivatives of $D$ glucopyranose. J Chem Society 1954; 1: 171-176.

26. Coimbra MA, Goncalves F, Barros AS, Delgadillo I. FTIR spectroscopy and chemometric analysis of white wine polysaccharide extracts. J Agric Food Chem 2002; 50: 3405-3411.

27. Macdonald J, Galley HF, Webster NR. Oxidative stress and gene expression in sepsis. J Agric Food Chem 2003; 90: 221-232.

28. Dahl MK, Richardson T. Photogeneration of superoxide anion in serum of bovine milk and in model systems containing riboflavin and amino acid. J Dairy Sci 1978; 61: 400-407. 